\title{
A novel Arabidopsis marker line that strongly labels uninucleate microspores and the subsequent male gametophyte development stages
}

\author{
José António da Costa-Nunes
}

\begin{abstract}
Here is reported a novel histological marker line that strongly and specifically labels the male gametophyte in Arabidopsis flowers, from the uninucleate microspore to the pollination stage. In this marker line, the expression of the UidA and GFP marker genes is driven by the promoter of the At5g17340 Arabidopsis gene, a gene highly expressed in the male gametophyte. Strong GUS histochemical staining is detected in the uninucleate microspores. Strong GUS, and GFP, histological labelling is also detected in the subsequent stages of male gametophyte development. The potential applications of this marker line are discussed.
\end{abstract}

Keywords: Microspore, Pollen, GUS, GFP, At5g17340, Marker line

\section{Introduction}

The Arabidopsis male gametophyte development is well characterised at the cytological level, and is a good system to study mitotic cell divisions. It is also a good system to indirectly characterise meiosis, since all four products of a single male meiotic cell division are viable, giving rise to microspores and pollen grains. The haploid unicellular uninucleate microspores undergo two rounds of mitotic divisions. The first mitotic cell division gives rise to bicellular pollen grains. As a result of the second mitotic cell division, tricellular pollen grains containing two gamete cells (the sperm cells) are formed. The bicellular pollen grain contains two nuclei, a condensed germinative nucleus and a large vegetative nucleus. The tricellular pollen contains a large vegetative nucleus and two condensed sperm nuclei (da Costa-Nunes and Grossniklaus 2003; Johnson-Brousseau and McCormick 2004; Borg et al. 2009). During pollination, the tricellular pollen grain germinates on the stigmatic papillae, producing a pollen tube that grows down the pistil and is attracted to one ovule (which holds the female gametophyte) (Shimizu and Okada 2000). While in wild-type

Correspondence: j.dacostanunes@wolfson.oxon.org

CBAA - Instituto Superior de Agronomia, Universidade Técnica de Lisboa, Tapada da Ajuda, Lisboa P-1349-017, Portugal
Arabidopsis, the microspores and pollen grains are individualised, in the quartet (qrt) mutants the four male meiotic products remain bound together in a tetrahedron (tetrad) configuration throughout the whole of gametophyte development, even during pollination. Hence the qrt mutations allow the scrutiny of the fate of all four products of a single meiotic cell division (Preuss et al. 1994).

To characterise male gametophyte development and pollination, several transgenic marker lines exhibiting $\beta$-glucuronidase induced (GUS) staining or green fluorescence protein (GFP) signal at different stages of the male gametophyte have been produced and used. One of the marker lines generally used in studies involving the male gametophyte, is the one carrying the pLAT52:UidA construct (Eady et al. 1995; Johnson et al. 2004). This construct, which contains a promoter from a tomato gene ( $p L A T 52)$, does not induce GUS staining in all Arabidopsis male gametophyte stages. It only induces GUS staining immediately before the first gametophytic mitotic division, and in later stages of the male gametophyte (in bicellular and tricellular pollen grains) (Eady et al. 1994). Likewise, the pLAT52:GFP marker line has been reported to induce fluorescence signal in pollen grains, as well as in pollen tubes (Palanivelu and Preuss 2006; Francis et al. 2007). 
Another Arabidopsis marker line that uses a nonArabidopsis promoter to induce strong and specific GUS staining in the male gametophyte, is the $p B n M 3.4: U i d A$ marker line. In this marker line, the Brassica napus promoter sequence ( $p B n M 3.4)$ induces GUS staining in Arabidopsis bicellular and tricellular pollen, yet, unlike pLAT52:UidA, it also induces strong GUS staining in Arabidopsis uninucleate microspores (Fourgoux-Nicol et al. 1999). Several Arabidopsis promoter sequences have also been shown to induce strong GUS staining in Arabidopsis flowers, in a male gametophyte and anther specific manner. However, these Arabidopsis promoters, either do not induce GUS staining in all stages of the male gametophyte, or if they do, they do not exhibit strong GUS staining at some of these stages (in particular in uninucleate microspores) (Moore et al. 1997; Li et al. 1998; Honys et al. 2006; Takeda et al. 2006; Gibalová et al. 2009; Phan et al. 2011).

Here is reported the production and characterisation of a novel marker line ( $p$ At5g17340:UidA:GFP) that uses an Arabidopsis promoter sequence to generate strong GUS staining in uninucleate microspores, as well as strong GUS staining and GFP signal in the subsequent pollen development stages, and hence it is a potentially good marker line to quickly and easily tag all stages of the Arabidopsis male gametophyte, including pollination.

\section{Methods}

\section{Plant material}

Arabidopsis thaliana landrace Columbia-0 (Col) (N1093) and the qrt mutant (qrt1-1; Landsberg erecta landrace; N8050) (Copenhaver et al. 1998) were obtained from the Nottingham Arabidopsis Stock Centre. Controlled crosspollination was used to combine the histological marker construct with the qrt1-1 mutation.

Prior to germination, seeds were sterilised and imbibed in the dark at $4^{\circ} \mathrm{C}$, for three to four days. Seeds were germinated in sterile Petri dishes containing solid germination medium (GM) (MS medium + Gamborg B5 vitamins, $1 \%$ sucrose; $0.8 \%$ micro-agar - Duchefa) or GM complemented with hygromycin. Alternatively, seeds were germinated in pots containing a sterilised commercial mix of turf, soil and fertiliser; pH 5.5 - 6.5. Seeds were germinated and plants grown in growth chambers with a cycle of 16 hours of light at $22^{\circ} \mathrm{C}$ alternating with 8 hours of darkness at $19^{\circ} \mathrm{C}$.

\section{Cloning and plant transformation}

A 2381 nucleotide base pairs (bp) PCR product containing the upstream region (henceforth promoter) of the At5g17340 gene, a gene highly expressed in uninucleate microspores (Honys and Twell 2004), was PCR amplified using Bio-X-ACT-TAQ-LONG (Bio-X-ACT) polymerase, the primers ${ }^{5}$ GTGTTTTTCAGAAGTCTATGAGCTC
ATTAG $^{3 \prime}$ and ${ }^{5}$ 'CTCCATGGTTGGATTTTTTAGGAAA CTTTTG $^{3}$, and wild-type Col-0 landrace genomic DNA as template; $\mathrm{SacI}$ and $\mathrm{NcoI}$ restriction sites were incorporated in the primers sequences (underlined nucleotides). The blunt-end PCR products were dATP tailed and cloned into the pGEMT-Easy (invitrogene) vector. The cloned inserts were excised with SacI and partial NcoI digest and cloned into the SacI/NcoI double digested pCAMBIA1303 binary vector (http://www.cambia.org/ daisy/cambia/585). Sequencing was used to verify the identity of the cloned inserts, and to certify that the insert was cloned in the correct reading frame.

Col-0 plants were transformed using the inflorescence dipping protocol (Clough and Bent 1998) and Agrobacterium tumefaciens (GV3101 strain) carrying the plasmid pMP90RK (Koncz and Schell 1986) and the binary vector construct. Transformants were selected on GM supplemented with hygromycin. Transformants (carrying the At5g17340 promoter construct) were genotyped using the GUSOUT1 ( ${ }^{5}$ GACTTCGCGCTGATACCAG $\left.\mathrm{ACG}^{3}\right)$ and P5 ( ${ }^{5^{\prime}}$ CATGCATAAGATATCGATATCAG $\left.{ }^{3}\right)$ primers; plant genomic DNA was extracted using the Edwards et al. (1991) protocol. Transformed Columbia-0 landrace plants were cross-pollinated with the qrt1-1 mutant (Landsberg erecta landrace) (Copenhaver et al. 1998). qrt1-1/qrt1-1 plants carrying the construct were identified on a screen for pollen with the qrt phenotype, on hygromycin resistant plants.

\section{Histochemical GUS staining and microscopy}

Histochemical GUS staining of inflorescences was carried out as described in Jefferson et al. (1987) and RodriguesPousada et al. (1993), with over-night incubation, at $37^{\circ} \mathrm{C}$, with X-Gluc (5-bromo-4-chloro-3-indolyl beta3-D-glucuronic acid, cyclohexylammonium salt, $\mathrm{MW}=$ 520.8 ) substract solution (2 $\mathrm{mg} / \mathrm{ml} \mathrm{X-Guc;} 50 \mathrm{mM} \mathrm{Na}$ Phosphate buffer, pH 7.0; 2 mM K ${ }_{3} \mathrm{Fe}(\mathrm{CN})_{6} ; 2 \mathrm{mM} \mathrm{K}_{4} \mathrm{Fe}$ $(\mathrm{CN})_{6} ; 10$ mM EDTA; 0.1\% Triton-X). After GUS staining incubation, the inflorescences were subjected to three consecutive ethanol washes (95\%, 80\%, 70\% ethanol). Intact anthers and dissected anthers from GUS stained and non-GUS stained flower buds were prepared for microscopy observation and mounted in a solution containing $2 \mu \mathrm{g} / \mathrm{ml}$ of DAPI (4',6-diamino-2-phenylindole, dihydrochloride). GFP was observed in freshly harvested microspores and pollen grains, or whole anthers, mounted in DAPI $(2 \mu \mathrm{g} / \mathrm{ml})$ solution. Observations were carried out in an epifluorescence and optical microscope (Zeiss Axioskop 2) and in a dissecting scope (Wild M3Z Magnifying scope); photographs were taken with an Axiocam (Zeiss) digital camera. Images were processed using the Photoshop and Microsoft Windows Paint programs. 


\section{Results}

To produce and obtain marker lines with strong and specific histological staining during the entire male gametophyte development, and in particular in the uninucleate microspores, promoter sequences of genes highly expressed in microspores were selected to drive the expression of the UidA and GFP markers genes. These two histological markers (GUS and GFP) were chosen because with these, simple and low cost male gametophyte specific histological labelling can be achieved and easily detected. The selection of the candidate genes (and hence, their promoters) was based on the data available from publications reporting gene expression in pollen, and other floral organs and somatic tissues (Honys and Twell 2004; Schmid et al. 2005; Winter et al. 2007).

\section{The At5g17340 promoter sequence promotes strong GUS} staining in anthers

One of the selected genes, the At5g17340, is a gene predicted to code for a plant specific 160 aminoacids transmembrane peptide. At5g17340 is the gene with the highest expression level in uninucleate microspores; it is also highly expressed in bicellular and tricellular pollen (Honys and Twell 2004). Its expression is reported to be restricted to the male gametophyte and anthers; it is not expressed in female gametophyte cells (synergids, egg cell, central cell) and it has no significant expression in other flower components (petals, sepals) (Schmid et al. 2005; Winter et al. 2007; Wuest et al. 2010). It is also not expressed during meiosis (Yang et al. 2011).
The amplified 2353 bp promoter sequence of the At5g17340 gene (pAt5g17340) was cloned in the pCAMBIA1303 binary vector to promote the simultaneous expression of the marker genes UidA (to induce GUS staining) and GFP. Transformation of Col-0 plants with the binary vector carrying the pAt5g17340:UidA:GFP construct, yielded 26 different independently transformed plants, 22 of which induce GUS staining in pollen. 15 of these lines induce GUS staining specifically in anthers and the male gametophyte. The GUS staining screen, focusing only on the flowers of these 15 lines, showed that GUS staining is detected in anthers (Additional file 1: Figure S1), in the tapetum (not shown) and the male gametophyte (Figure 1). It also showed that the anther specific GUS staining is developmentally dependent; it is absent in younger (smaller) flowers and it is present in anthers of older (bigger) flowers (Additional file 1: Figure S1b-e). 6 out of the 15 screened lines, also strongly GUS stain petals, sepals and carpels, after overnight incubation with X-Gluc (data not shown). Some of the lines with an anther specific GUS staining pattern also weakly stain the papillae. No GUS staining is detected in wild-type inflorescence after over-night incubation with X-Gluc (Additional file 1: Figure S1a).

\section{Strong GUS staining is detected in the male gametophyte, from the uninucleate microspore stage to pollen tube germination}

Further characterisation of the marker line was carried out in 8 of the plant lines exhibiting strong anther specific

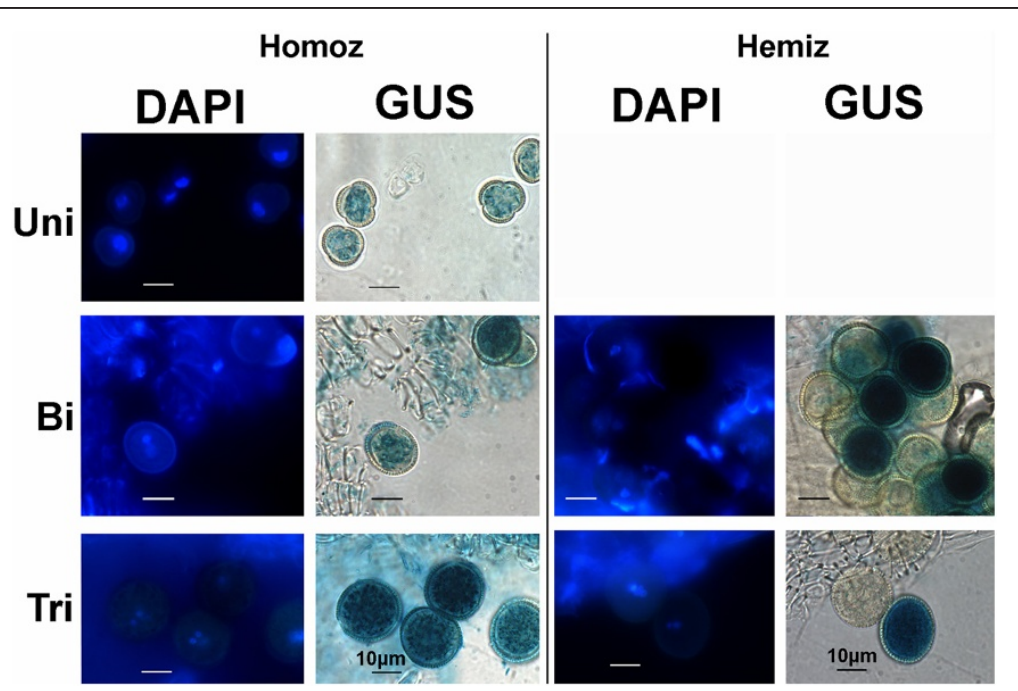

Figure 1 Strong GUS staining in uninucleate microspores and pollen grains of the pAt5g17340:UidA:GFP marker line. Strong GUS staining is detected in late uninucleate microspores (Uni), as well as in bicellular (Bi) and tricellular pollen (Tri) of plants homozygous for the pAt5g17340:UidA:GFP construct. Segregation of the marker gene (GUS stained and non-GUS stained pollen in a 1:1 ratio), as illustrated in the bicellular (Figure 1-Bi-Hemiz) and tricellular (Figure 1-Tri-Hemiz) pollen grains, indicates that the hemizygous plant has a single UidA marker gene insertion in the genome. Plates show DAPI stained nuclei (left column) of the GUS stained (right column) microspores and pollen grains in homozygous (Homoz) and hemizygous (Hemiz) plants. Uni- uninucleate microspore; Bi- bicellular pollen; Tri- tricellular pollen. Homozygous marker line plant: G63; Hemizygous marker line plant: GC3. GUS staining obtained after overnight incubation. Scale bars correspond to $10 \mu$ m. 
GUS staining. Very weak GUS staining can be first detected in microspores at the early microspore stage (early uninucleate microspore) (Additional file 2: Figure S2a). No GUS staining was detected in flowers at earlier developmental stages (data not shown). Later in development, strong GUS staining is detected in uninucleate microspores (late uninucleate microspores) (Figure 1-Uni; Additional file 2: Figure S2b), as well as in bicellular (Figure 1-Bi) and tricellular pollen grains (Figure 1-Tri). In lines homozygous (Homoz) for the marker gene, all pollen grains are GUS stained (Figure 1-Homoz); hemizygous (Hemiz) plants contain GUS stained and non-GUS stained pollen grains, and exhibit a 1:1 segregation ratio of the marker gene in the male gametophyte, as illustrated in bicellular and tricellular pollen grains (Figure 1-Bi-Hemiz and 1-Tri-Hemiz). Col-0 flowers incubated with X-Gluc do not exhibit any GUS staining in the male gametophyte (Figure 2).

GUS staining, in the marker line, is also detected in germinating pollen grains attached to the stigmatic papillae (Figure 3a, white arrowheads) and in the pollen tubes growing down the stigma (Figure 3a, black arrow).
Selected plants of the pAt5g17340:UidA:GFP marker line were crossed to the qrt1-1 mutant (Copenhaver et al. 1998). In this new lines (pAt5g17340:UidA:GFP / qrt1-1/ qrt1-1), strong GUS staining is detected in the tetrads of pollen grains (Figure 3b, 3c), in contrast with the lack of GUS staining (after incubation with X-Gluc substract) in the tetrad of pollen grains of a qrt1-1 mutant lacking the marker gene (Figure 3d, 3e). This pAt5g17340:UidA:GFP / qrt1-1/qrt1-1 line was produced with the aim of aiding the characterisation of mutations affecting the male gametophyte or male meiosis.

\section{GFP signal is easily detected in bicellular and tricellular pollen grains}

No unambiguous GFP fluorescence signal is detected in uninucleate microspores (Figure 4-Uni) of the $p A t 5 g$ 17340:UidA:GFP marker line, in contrast with the strong GUS staining detected at this stage (Figure 1-Uni). In bicellular and tricellular pollen, however, strong GFP signal (Figure 4-Bi and 4-Tri) is detected; a signal that is clearly stronger than the pollen auto-fluorescence (Johnson-Brousseau and McCormick 2004). The segre-

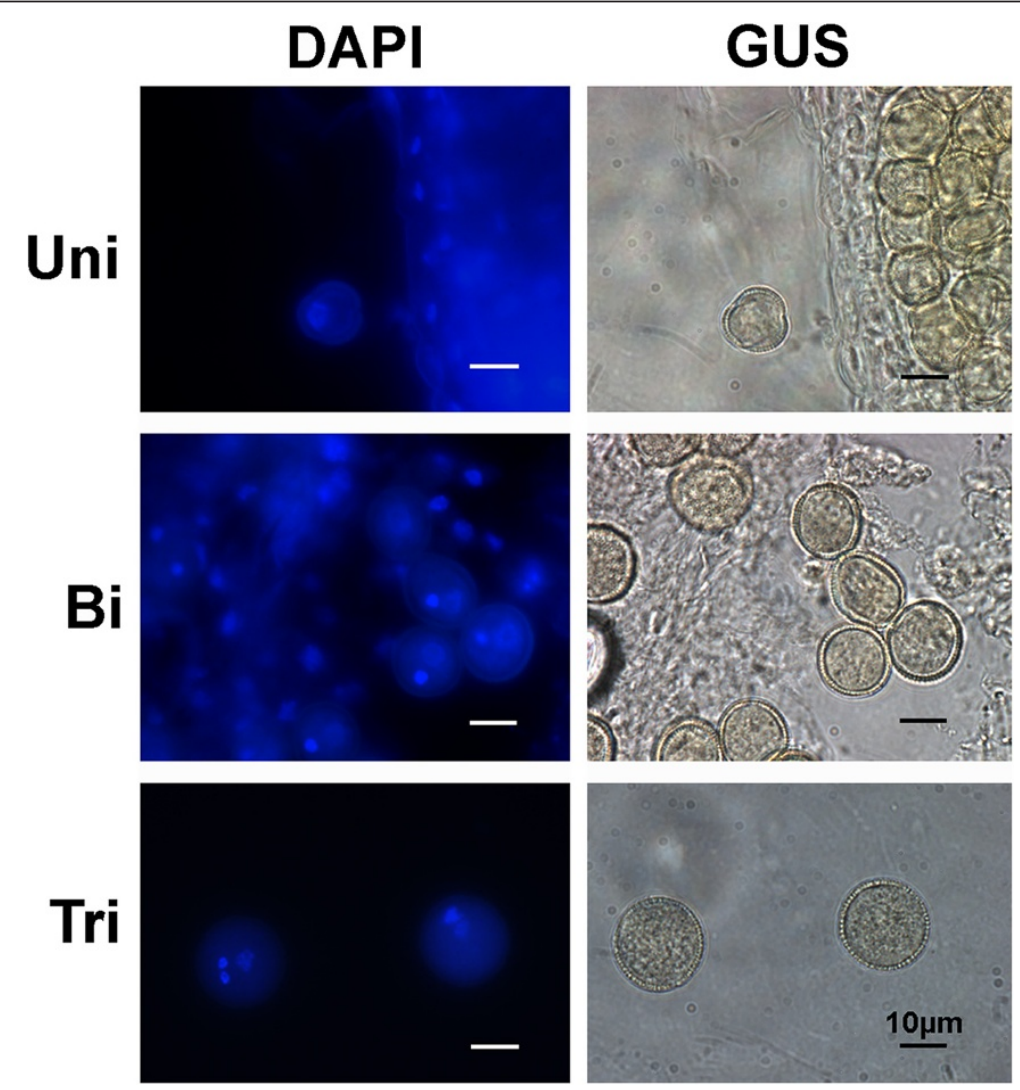

Figure 2 X-Gluc incubated Col-0 uninucleate microspores and pollen grains. No GUS staining (right column) is detected in late uninucleate microspores (Uni), nor in the bicellular (Bi) or tricellular (Tri) pollen grains of wild-type Col-0 landrace. The inflorescences were incubated overnight with X-Gluc substract to induce GUS staining. Plates show DAPI stained nuclei (left column) of the X-Gluc incubated (right column) microspores and pollen grains. Uni- uninucleate microspore; Bi- bicellular pollen; Tri- tricellular pollen. Col-0 plant. Scale bars correspond to $10 \mu \mathrm{m}$. 


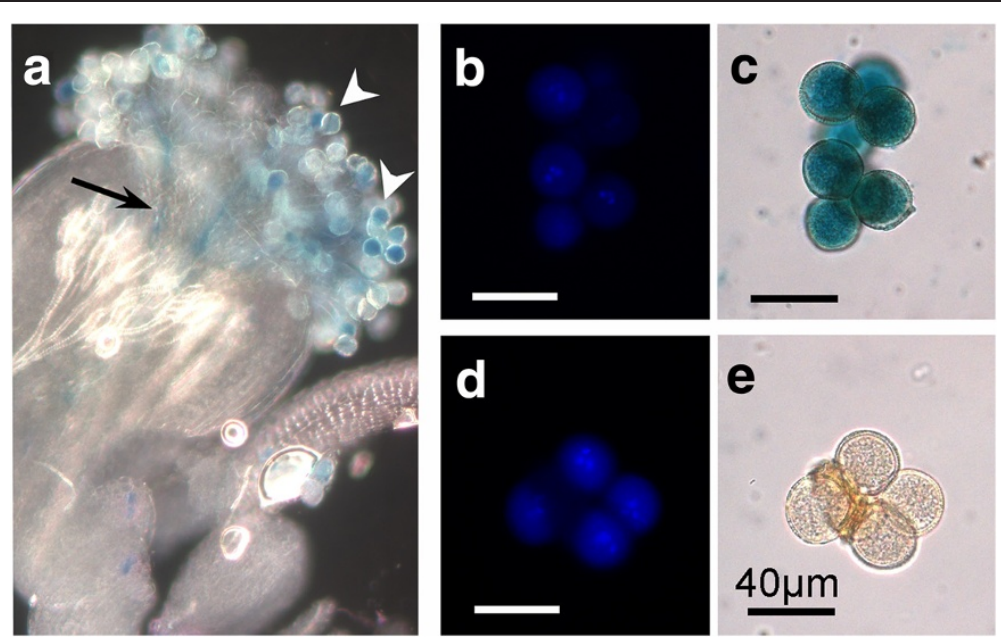

Figure 3 pAt5g17340:UidA:GFP induced GUS staining in qrt mutant pollen and during pollination. GUS staining is also detected in the germinating pollen grains (white arrowheads) and in the germinated pollen tube inside the style (black arrow) (a). GUS stained tetrads of tricellular pollen grains from the pAt5917340:UidA:GFP / grt1-1/qrt1-1 marker line (c) and tetrad of qrt1-1/qrt1-1 mutant tricellular pollen grains carrying no marker gene (e). DAPI staining (b, d) of the X-Gluc incubated pollen grains (c, e). GUS staining obtained after overnight incubation. Marker line plants: art1-1 and GC30 (with art1-1 pollen); G57 (pollination). Scale bars correspond to $40 \mu \mathrm{m}$.

gation of the GFP marker gene is also clearly and easily detected, in a 1:1 ratio (GFP:no-GFP), in the gametophyte of hemizygous plants (Figure 4-Bi, 4-Tri). This same GFP pattern (in the male gametophyte) is detected in this line (G65) as well as in others (GC7 - data not shown).

The construct that induces GFP expression in this line (G65; Figure 4), also induces strong anther specific and developmentally regulated GUS staining in the G138 plants (plants obtained from G65 self-pollination; Additional file 1: Figure S1). Hence, the same line can be used for both GUS staining and GFP labeling of the male gametophyte. At least, two more independent lines of the pAt5g17340:UidA:GFP marker line have been shown to provide both GFP labeling and GUS staining (data not shown).

Figure 4-Tri-Col shows wild-type (Col-0) tricellular pollen grains lacking GFP fluorescence signal.

Further information concerning the plant lines described in this report is provided in the Additional file 3: Table S1.

\section{Discussion}

The aim of the work reported here was to obtain marker lines that induce strong histological labelling (GUS or GFP) in all stages of the male gametophyte. The ultimate goal would be to use these lines in the characterisation of the male gametophyte of male-fertility-impaired mutants. Since many of these mutants produce aborted gametophytic cells (Johnson et al. 2004; Takeda et al. 2006; da Costa-Nunes and Viegas 2009; Gibalová et al. 2009), it is important to induce GUS staining before they collapse, otherwise it becomes difficult or impossible to detect GUS staining in these mutant gametophytic cells
(Johnson et al. 2004). Therefore, the pAt5g17340:UidA: GFP marker line was produced to strongly label the male gametophyte, as early as possible, in uninucleate microspores (Figure 1-Uni), as well as the subsequent stages of male gametophyte development.

pAt5g17340:UidA:GFP is a good marker line to induce strong male gametophyte specific GUS staining, in uninucleate microspores and pollen grains

In this report, it is shown that the flowers from the new pAt5g17340:UidA:GFP marker line plants exhibit strong and anther specific GUS staining (Additional file 1: Figure S1b-e), in uninucleate microspores and bicellular and tricellular pollen grains, after incubation with X-Gluc (Figure 1; Additional file 2: Figure S2). GUS staining is first detected in anthers accommodating uninucleate microspores (Additional file 2: Figure S2a), which indicates that the pAt5g17340:UidA:GFP construct does not induce GUS staining in anthers of flowers at the meiotic and premeiotic stage.

Of the several reported marker lines that use an Arabidopsis gene promoter to induce strong GUS staining in the male gametophyte, pAt3g45150:UidA is the only marker line that has been shown to induce particularly strong GUS staining in uninucleate microspores (Takeda et al. 2006). The intensity of the reported GUS staining is comparable to the one detected in the uninucleate microspores of the pAt5g17340:UidA:GFP marker line (Figure 1Uni). Yet, unlike pAt5g17340:LidA:GFP, the pAt3g45150: UidA does not induce GUS staining at the last stages of pollen development (Takeda et al. 2006). 


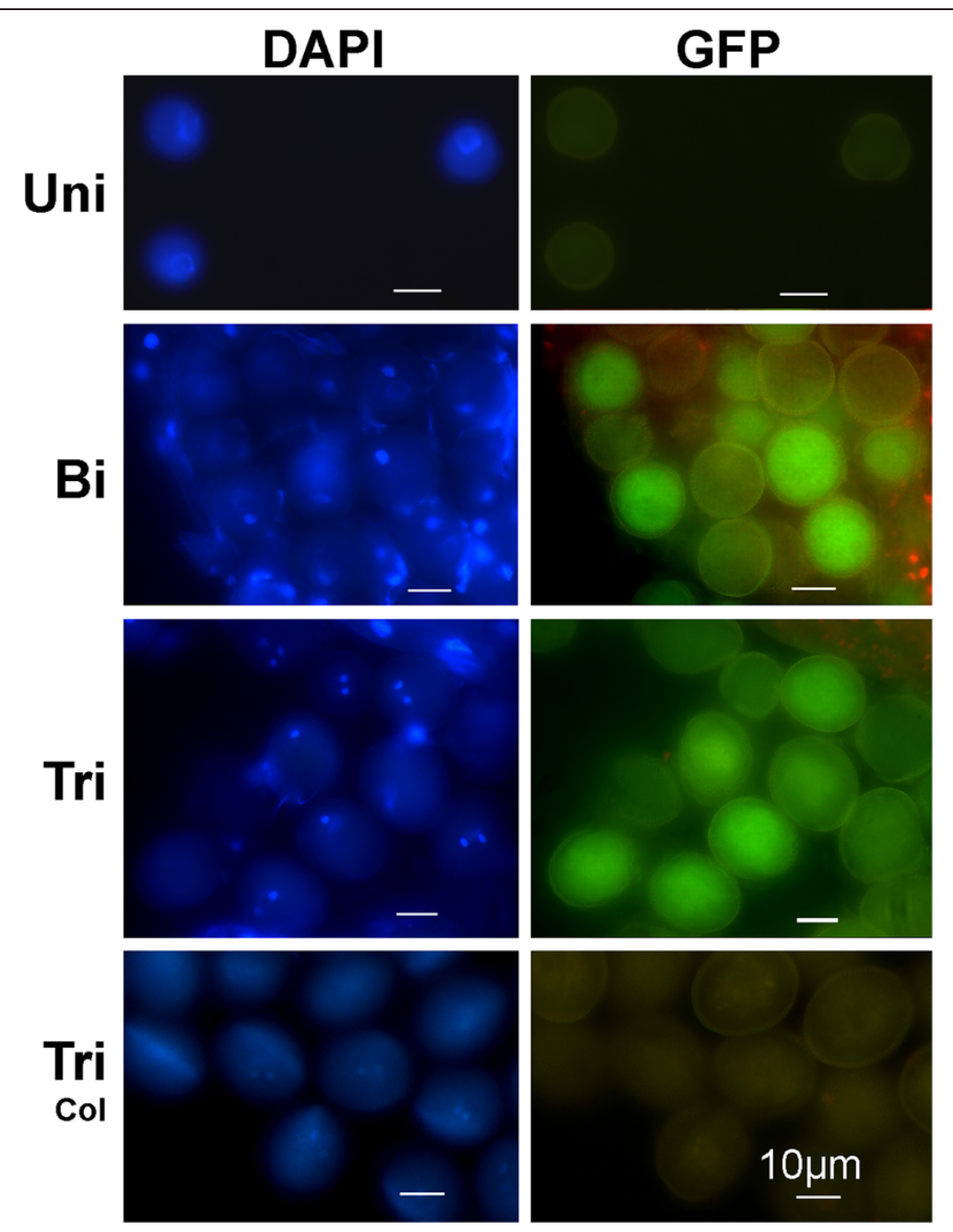

Figure 4 GFP labelling in pollen grains of the pAt5g17340:UidA:GFP marker line. GFP signal is clearly detected in bicellular (Bi) and tricellular (Tri) pollen grains of the pAt5g17340:UidA:GFP marker line. At the late uninucleate microspore stage (Uni), the pAt5g17340:UidA:GFP marker line GFP signal is very weak or undetectable. Segregation of the GFP signal (GFP versus non GFP - 1:1 ratio) is detected in the bicellular (Bi) and tricellular (Tri) pollen of a plant hemizygous for the GFP marker gene, indicating a single marker gene insertion in the genome. No GFP signal is detected in Col-0 tricellular pollen grains (Tri-Col). Plates show DAPI stained nuclei (left column) of the microspores and pollen grains shown in the right column. Uni- uninucleate microspore; Bi- bicellular pollen; Tri- tricellular pollen; Tri-Col- wild-type Col-0 tricellular pollen. Marker line plant: G65. Scale bars correspond to $10 \mu \mathrm{m}$.

Like pAt5g17340:UidA:GFP, there are other marker lines that use Arabidopsis promoters to induce convincing GUS staining in all the male gametophytic stages (Honys et al. 2006), yet the reported staining intensity in their uninucleate microspores is not as strong as in the pAt5g17340:UidA:GFP marker line.

In conclusion, the GUS staining data indicates that the novel marker line (pAt5g17340:UidA:GFP) is a good tool to characterise all stages of male gametophyte development. The pAt5g17340:UidA:GFP marker line is particularly good to characterise uninucleate microspores (Figure 1), and it can also be used to characterise pollen tube germination and pollination (Figure 3a). Furthermore, the data indicates that the GUS staining intensity and pattern observed in the pAt5g17340:UidA:GFP marker line (Figure 1) is coherent with the At5g17340 reported male gametophyte gene expression microarray data (Honys and Twell 2004) that indicates that this gene is expressed in high levels in uninucleate microspores, bicellular and tricellular pollen grains.

The promoter sequences of At5g17340 and its Brassica napus homologous gene share the ability to induce strong histochemical staining during the entire male gametophyte

There is one other Arabidopsis marker line, that like the pAt5g17340:UidA:GFP marker line, has been reported to induce strong GUS staining in uninucleate microspores, 
and in bicellular and tricellular pollen grains (FourgouxNicol et al. 1999). In this Arabidopsis marker line (pBnM3.4:UidA), the promoter (pBnM3.4) of the BnM3.4/ CL1Contig2422 Brassica napus gene is used to induce strong anther specific GUS staining. BnM3.4 and At5g17340 are homologous genes (Fourgoux-Nicol et al. 1999; Whittle et al. 2010). Both genes are expressed at high levels; BnM3.4 is the Brassica napus gene with the third highest expression level in Brassica napus microspores and early bicellular pollen (Whittle et al. 2010) while At5g17340 is the gene with the highest expression level in Arabidopsis uninucleate microspores (Honys and Twell 2004). The strong GUS staining obtained in the pAt5g17340:UidA:GFP and pBnM3.4:UidA marker lines is consistent with these high levels of gene expression. The GUS (or GFP) staining pattern also indicates, in particular in hemizygous plants segregating GUS stained and nonGUS stained (or GFP) pollen grains (Figures 1-Bi-Hemiz, 1-Tri-Hemiz, 4-Bi and 4-Tri) (Fourgoux-Nicol et al. 1999), that these homologous genes are gametophytically expressed. Hence, the promoters of the At5g17340 and $B n M 3.4$ homologous genes share and conserve the ability to induce high levels of gene expression (particularly in uninucleate microspores), male gametophytic specific gene expression, and similar and strong GUS staining patterns.

\section{pAt5g17340:UidA:GFP induced strong GFP fluorescence is suitable to characterise bicellular and tricellular pollen grains}

GFP signal (Figure 4) is clearly detected in plants carrying the pAt5g17340:UidA:GFP construct in both bicellular and tricellular pollen grains, yet no clear positive GFP fluorescence signal was observed in uninucleate microspores (Figure 4-Uni); It is not known why the GFP signal is not detected, with epifluorescence microscopes, in uninucleate microspores of this marker line. Hence, this marker line, like other previously reported marker lines such as the pLAT52:GFP (Francis et al. 2007), is only suitable to characterise (with GFP) the later stages of pollen development.

\section{The pAt5g17340:UidA:GFP / qrt1-1/qrt1-1 marker line}

In this line, that combines the pAt5g17340:UidA:GFP construct with the qrt1-1 mutation, the products of male meiosis are held together during the entire male gametophyte development. This line was produced to facilitate the characterisation and scrutiny of the fate, at all stages of male gametophyte development, of all meiotic products obtained from each single abnormal mutant meiotic cell division as well as of the microspores and pollen grains formed in gametophytic mutants. The strong GUS staining in uninucleate microspores (Figure 1-Uni), combined with the qrt1-1 mutation (Figure 3c), has potential interest for the characterisation of meiotic mutants, or gametophytic mutants, that produce aborted and collapsed gametophytic cells at the early stages of male gametophyte development.

\section{Additional files}

\begin{abstract}
Additional file 1: Figure S1. GUS staining in the inflorescence is anther specific and developmentally regulated. Inflorescence side view (a, b, d, e) and top view (c) showing GUS staining only in the anthers of the older (outer) flowers of different independently obtained transformed lines ( $b, c, d, e)$ of the pAt5g17340:UidA:GFP marker line. No GUS staining is detected in the inner (younger) flowers of the inflorescence from different independent lines from this marker line $(b, c, d, e)$ nor in the wild-type Col-0 inflorescence (a). White arrow points to the smaller (younger) flower buds where GUS staining is first detected (e). Marker line plants: GC21 (b, c); G138 (d) and G57 (e) are descendent from three different independently transformed lines; line G138 is descendent from the self-pollinated G65 line (see Figure 4); self-pollination of G57 (see also Additional file 2: Figure S2) gave origin to the line G63 (see Figure 1). Col-0 (a). GUS staining obtained after overnight incubation.

Additional file 2: Figure S2. GUS staining in flowers at different developmental stages, and the respective uninucleate microspores and pollen grains. All flowers were harvested from a single inflorescence of a pAt5g17340:UidA:GFP marker line plant. $a, b$ - Young flowers with GUS stained anthers, containing uninucleate microspores; a - early uninucleate microspores with weak GUS staining; $b$ - late uninucleate microspore with strong GUS staining. Flowers smaller than the one depicted in plate ' $a$ ' did not exhibit GUS staining (not shown). c, d, e, f - Flowers with anthers containing GUS stained bicellular (c, d) and tricellular (e) pollen grains. Neither DAPI nor GUS stained pollen grains from the flower ' $f$ ' are shown. GUS stained pollen tube elongating in the pistil is detected in plate ' $e$ ' (Flower). $f$ - An older flower with GUS stained pollen grains attached to the stigma's papillae and inside the dehiscent anthers. The microspores and pollen grains that are shown were dissected from one or two anthers removed from the flowers depicted in the (Flower) plates. Flower plates - Whole flower with GUS stained anthers. DAPI plates show the DAPI stained nuclei of the GUS stained microspores and pollen grains (GUS plates). Marker line plant: G57. GUS staining obtained after overnight incubation. Scale bars correspond to $0.4 \mathrm{~mm}$ in Flower plates, and to $15 \mu \mathrm{m}$ in DAPI and GUS plates.
\end{abstract}

Additional file 3: Table S1. The different (and independently transformed) lines from the pAt5g17340:UidA:GFP marker line reported in this manuscript. Table listing the lines reported in this manuscript.

\section{Competing interest}

The author declares that there is no competing interest.

\section{Acknowledgements}

JA da C-N is thankful to the Fundação para a Ciência e a Tecnologia (FCT) (fellowships SFRH/BPD/7137/2001 and SFRH/BPD/30365/2006) and Prof. W. Viegas (Universidade Técnica de Lisboa, Portugal) who provided facilities and resources to carry out the work in her laboratory. JA da C-N is grateful to Olga Pontes (University of New Mexico, USA) for critical reading of the manuscript.

Received: 14 February 2013 Accepted: 16 May 2013 Published: 24 May 2013

\section{References}

Borg M, Brownfield L, Twell D (2009) Male gametophyte development: a molecular perspective. J Exp Bot 60:1465-1478

Clough SJ, Bent AF (1998) Floral dip: a simplified method for Agrobacteriummediated transformation of Arabidopsis thaliana. Plant J 16:735-743

Copenhaver GP, Browne WE, Preuss D (1998) Assaying genome-wide recombination and centromere functions with Arabidopsis tetrads. Proc Natl Acad Sci USA 95:247-252 
Da Costa-Nunes JA, Grossniklaus U (2003) Unveiling the gene-expression profile of pollen. Genome Biol 5:205

Da Costa-Nunes JA, Viegas W (2009) An approach to screen and identify novel meiotic mutants in an EMS mutant population. In: Shu QY (ed) Induced Plant Mutations in the Genomics Era. Food and Agriculture Organization (F.A.O.) of the United Nations, Rome, pp 126-128

Eady C, Lindsey K, Twell D (1994) Differential activation and conserved vegetative cell-specific activity of a late pollen promoter in species with bi- and tricellular pollen. Plant J 5:543-550

Eady C, Lindsey K, Twell D (1995) The significance of microspores division and division symmetry for vegetative cell-specific transcription and generative cell differentiation. Plant Cell 7:65-74

Edwards K, Johnstone C, Thompson C (1991) A simple and rapid method for the preparation of plant genomic DNA for PCR analysis. Nucleic Acids Res 19:1349

Fourgoux-Nicol A, Drouaud J, Haouazine N, Pelletier G, Guerche P (1999) Isolation of rapeseed genes expressed early and specifically during development of the male gametophyte. Plant Mol Biol 40:857-872

Francis KE, Lam SY, Harrison BD, Bey AL, Berchowitz LE, Copenhaver GP (2007) Pollen tetrad-based visual assay for meiotic recombination in Arabidopsis. Proc Natl Acad Sci USA 104:3913-3918

Gibalová A, Reňák D, Matczuk K, Dupl'áková N, Cháb D, Twell D, Honys D (2009) AtbZIP34 is required for Arabidopsis pollen wall patterning and the control of several metabolic pathways in developing pollen. Plant Mol Biol 70:581-601

Honys D, Twell D (2004) Transcriptome analysis of haploid male gametophyte development in Arabidopsis. Genome Biol 5:R85

Honys D, Oh SA, Reňak D, Donders M, Šolcová B, Johnson JA, Boudová R, Twell D (2006) Identification of microspore-active promoters that allow targeted manipulation of gene expression at early stages of microgametogenesis in Arabidopsis. BMC Plant Biol 6:31-39

Jefferson RA, Kavanagh TA, Bevan MW (1987) GUS fusions: $\beta$-glucuronidase as a sensitive and versatile gene fusion marker in higher plants. EMBO J 6:3901-3907

Johnson MA, Von Besser K, Zhou Q, Smith E, Aux G, Patton D, Levin JZ, Preuss D (2004) Arabidopsis hapless mutations define essential gametophytic functions. Genetics 168:971-982

Johnson-Brousseau SA, McCormick S (2004) A compendium of methods useful for characterizing Arabidopsis pollen mutants and gametophyticallyexpressed genes. Plant J 39:761-775

Koncz C, Schell J (1986) The promoter of $T_{1}-D N A$ gene 5 controls the tissuespecific expression of chimaeric genes carried by a novel type of Agrobacterium binary vector. Mol Gen Genet 204:383-396

Li H, Wu G, Ware D, Davis KR, Yang Z (1998) Arabidopsis Rho-related GTPases: differential gene expression in pollen and polar localization in fission yeast. Plant Physiol 118:407-417

Moore I, Diefenthal T, Zarsky V, Schell J, Palme K (1997) A homolog of the mammalian GTPase Rab2 is present in Arabidopsis and is expressed predominantly in pollen grains and seedlings. Proc Natl Acad Sci USA 94:762-767

Palanivelu R, Preuss D (2006) Distinct short-range ovule signals attract or repel Arabidopsis thaliana pollen tubes in vitro. BMC Plant Biol 6:7

Phan HA, lacuone S, Li SF, Parish RW (2011) The MYB80 transcription factor is required for pollen development and the regulation of tapetal programmed cell death in Arabidopsis thaliana. Plant Cell 23:2209-2224

Preuss D, Rhee SY, Davis RW (1994) Tetrad analysis possible in Arabidopsis with mutation of the Quartet (QRT) gene. Science 264:1458-1460

Rodrigues-Pousada RA, De Rycke R, Dedonder A, Caeneghem WV, Engler G, Van Montagu M, Van Der Straeten D (1993) The Arabidopsis 1aminocyclopropane-1-carboxylate synthase gene 1 is expressed during early development. Plant Cell 5:897-911

Schmid M, Davison TS, Henz SR, Pape UJ, Demar M, Vingron M, Schölkopf B, Weigel D, Lohmann JU (2005) A gene expression map of Arabidopsis thaliana development. Nat Genet 37:501-506

Shimizu KK, Okada K (2000) Attractive and repulsive interactions between female and male gametophytes in Arabidopsis pollen tube guidance. Development 127:4511-4518

Takeda T, Amano K, Ohto M-A, Nakamura K, Sato S, Kato T, Tabata S, Uegushi C (2006) RNA interference of the Arabidopsis putative transcription factor TCP16 gene results in abortion of early pollen development. Plant Mol Biol 61:165-177
Whittle CA, Malik MR, Li R, Krochko JE (2010) Comparative transcript analyses of the ovule, microspore, and mature pollen in Brassica napus. Plant Mol Biol 72:279-299

Winter D, Vinegar B, Nahal H, Ammar R, Wilson GV, Provart NJ (2007) An "electronic fluorescent pictograph" browser for exploring and analyzing large-scale biological data sets. PLoS One 2:e718

Wuest SE, Vijverberg K, Schmidt A, Weiss M, Gheyselinck J, Lohr M, Wellmer F, Rahnenführer J, Von Mering C, Grossniklaus U (2010) Arabidopsis female gametophyte gene expression map reveals similarities between plant and animal gametes. Curr Biol 20:506-512

Yang H, Lu P, Wang Y, Ma H (2011) The transcriptome landscape of Arabidopsis male meiocytes from high-throughput sequencing: the complexity and evolution of the meiotic process. Plant J 65:503-516

doi:10.1186/2193-1801-2-237

Cite this article as: da Costa-Nunes: A novel Arabidopsis marker line that strongly labels uninucleate microspores and the subsequent male gametophyte development stages. SpringerPlus 2013 2:237.

\section{Submit your manuscript to a SpringerOpen ${ }^{\circ}$ journal and benefit from:}

- Convenient online submission

- Rigorous peer review

- Immediate publication on acceptance

- Open access: articles freely available online

High visibility within the field

- Retaining the copyright to your article

Submit your next manuscript at $\gg$ springeropen.com 